\title{
Computing the Forcing and Anti-Forcing Numbers of Perfect Matchings for Graphs by Integer Linear Programmings*
}

\author{
Yutong Liu, Congcong Ma, Haiyuan Yao ${ }^{\dagger}, \mathrm{Xu}$ Wang \\ College of Mathematics and Statistics, Northwest Normal University, \\ Lanzhou, Gansu 730070, P. R. China \\ 2315631706@qq.com; 15117076629@163.com; hyyao@nwnu.edu.cn; \\ cwangx@hotmail.com
}

(Received July 12, 2021)

\begin{abstract}
The forcing polynomial and anti-forcing polynomial are two important enumerative polynomials associated with all perfect matchings of a graph. In a graph with large order, the exhaustive enumeration which is used to compute forcing number of a given perfect matching is too time-consuming to compute anti-forcing number. In this paper, we come up with an efficient method - integer linear programming, to compute forcing number and anti-forcing number of a given perfect matching. As applications, we obtain the di-forcing polynomials $C_{60}, C_{70}$ and $C_{72}$, and as a consequence, the forcing and anti-forcing polynomials of them are obtained.
\end{abstract}

\section{Introduction}

All graphs considered in this paper are finite and simple. A perfect matching or 1-factor $M$ of a graph $G$ is a set of independent edges incidenting with every vertices of $G$, which is also called a Kekulé structure in chemical literatures. In 1991, the forcing numbers for perfect matchings of graphs are proposed by F. Harary, D. Klein and T. Živkovič [10] while studying the hexagonal system, which are equivalent to the chemical concept innate degrees of freedom of Kekulé structures, given by D. Klein and M. Randić $[13,19]$ in study of the molecular resonance structures. In 2004, the forcing spectrum, the set

\footnotetext{
*Supported by grants from The National Nutural Science Foundation of China (No. 12161081).

†Corresponding author.
} 
of forcing numbers of all perfect matchings, of $G$ is defined by P. Adams, M. Mahdian and E. Mahmoodian [3,4]. For more researches on forcing sets and forcing numbers, the interested readers may refer to a recent survey [5] and references therein. In oder to refine forcing spectrum, H. Zhang, S. Zhao and R. Lin [29] proposed the concept of the forcing polynomial.

As a "complement" of innate degrees of freedom (also known as $e$-freedom [14]) of Kekulé structures, D. Klein proposed the (e)-freedoms of Kekulé structures [14], which was independently proposed by H. Lei, Y. Yeh and H. Zhang [16], under the name of anti-forcing numbers of Kekulé structures. In 2007, D. Vukičević and N. Trinajstić [26] proposed the concept of the anti-forcing number of a graph, that is the minimum antiforcing number of all perfect matchings of the graph. In fact, early in 1997, X. Li [18] had studied the hexagonal system $H$ with a forcing single edge $e$ (now called an anti-forcing edge), i.e., $\{e\}$ is an anti-forcing set of $H$. Analogous to the forcing polynomial, H. Hwang, H. Lei, Y. Yeh and H. Zhang [11] introduced the concept of an enumerative polynomial — the anti-forcing polynomial for a graph.

The forcing polynomials and the anti-forcing polynomials of some graphs had been obtained. K. Deng, S. Liu, X. Zhou, H. Lü and T. Wu [7,8] studied forcing and anti-forcing polynomials of a pyrene system and a polyomino graph. S. Zhao and H. Zhang obtained the recursive relation of forcing polynomials of cata-condensed hexagonal systems [29], the forcing polynomials for benzenoid parallelograms [30], anti-forcing polynomials of phenyl systems [30], and anti-forcing polynomials of $P_{2} \times P_{n}$ rectangle grids and $P_{3} \times P_{2 n}$ rectangle grids [31]. Although the forcing polynomials of Fullerene graphs $C_{60}, C_{70}, C_{72}$ have been obtained $[20,22,23]$, the anti-forcing polynomials of them are still not computed.

The Clar and Fries numbers are two important indicators relevant to perfect matchings of benzenoid graphs [6,9]. P. Hansen and M. Zheng [17] formulated the Clar number of a graph as an integer programming. H. Abeledo and G.W. Atkinson [1,2] substantiated that the linear relaxation of the integer programming has integer optimal solution by proving that the constraint matrix of the Clar integer programming is invariably totally unimodular; they also formulated the Fries number as an integer programming and obtained a similar result. Thus, the Clar and Fries numbers can be computed in polynomial time using linear programmings. In this paper, we also formulate the forcing and anti-forcing numbers of a perfect matchimg as an integer linear programmings. However, the linear 
relaxations of them may have fractional optimal solutions (see Remark 1(3)).

The structure of this paper is as follows. In Section 2, we introduce some basic terminologies, symbols, conclusions and the main concept - di-forcing polynomial. In Section 3, we introduce the exhaustive enumeration and come up with the integer linear programming to compute forcing and anti-forcing numbers of a given perfect matching. In Section 4, we use integer linear programming to compute di-forcing polynomials of $C_{60}$, $C_{70}$ and $C_{72}$, and as a direct application, the forcing and anti-forcing polynomials of them are obtained.

\section{Preliminaries}

Let $G=(V, E)$ be a simple graph with a perfect matching $M$. A cycle of $G$ is $M$ alternating if its edges are alternately in and out of $M$.

A subset $S \subseteq M$ is a forcing set of a perfect matching $M$ if $M$ is the unique perfect matching of $G$ which contains $S$. The forcing number of $M$ is the smallest cardinality over all forcing sets of $M$, denoted by $f(G, M)$. The minimum (resp. maximum) forcing number of $G$ is the minimum (resp. maximum) value of forcing numbers of all perfect matchings of $G$, denoted by $f(G)$ (resp. $F(G)$ ). In the early literatures, $f(G)$ is also known as the forcing number of $G$.

A subset $S_{a} \subseteq E \backslash M$ is an anti-forcing set of $M$ if $G \backslash S_{a}$ has the unique perfect matching $M$, where $G \backslash S_{a}$ denotes the resulting subgraph obtained from $G$ by deleting the edges in $S_{a}$. The anti-forcing number of $M$ is the smallest cardinality over all anti-forcing sets of $M$, denoted by af $(G, M)$. The minimum (resp. maximum) anti-forcing number of $G$ is the minimum (resp. maximum) value of anti-forcing numbers of all perfect matchings of $G$, denoted by $a f(G)$ (resp. $A f(G)$ ). Similarly, af $(G)$ is also known as the name of the anti-forcing number of $G$.

Here is a characterization of a forcing set.

Lemma 2.1 [3,21] Let $M$ be a perfect matching of a graph $G$. A subset $S \subseteq M$ is a forcing set of $M$ if and only if $S$ contains at least one edge of each $M$-alternating cycle.

There is a similar characterization of the anti-forcing set. 
Lemma 2.2 [16] Let $M$ be a perfect matching of a graph $G$. A subset $S_{a} \subseteq E \backslash M$ is an anti-forcing set of $M$ if and only if $S_{a}$ contains at least one edge of each $M$-alternating cycle.

The forcing and anti-forcing polynomials are enumerative polynomials w.r.t. forcing and anti-forcing numbers of all perfect matchings of $G$, respectively. Let $\mathcal{M}(G)$ be the set of all perfect matchings of $G$.

Definition 2.1 [11,29] The forcing polynomial of $G$ is

$$
F(G, x)=\sum_{M \in \mathcal{M}(G)} x^{f(G, M)}=\sum_{i=f(G)}^{F(G)} \omega(G, i) x^{i},
$$

where $\omega(G, i)$ refers to the number of perfect matchings of $G$ with forcing number $i$.

Definition 2.2 [11,30] The anti-forcing polynomial of $G$ is

$$
A f(G, x)=\sum_{M \in \mathcal{M}(G)} x^{a f(G, M)}=\sum_{i=a f(G)}^{A f(G)} \mu(G, i) x^{i},
$$

where $\mu(G, i)$ refers to the number of perfect matchings of $G$ with anti-forcing number $i$.

Observing a striking similarity between the above two definitions, we naturally combine these two polynomials into a single two-variable polynomial - di-forcing polynomial.

Definition 2.3 Let $G$ be a graph. The di-forcing polynomial of $G$ is

$$
F a f(G ; x, y)=\sum_{M \in \mathcal{M}(G)} x^{f(G, M)} y^{a f(G, M)}=\sum_{i, j} \nu(G ; i, j) x^{i} y^{j},
$$

where $\nu(G ; i, j)$ refers to the number of perfect matchings of $G$ with forcing number $i$ and anti-forcing number $j$.

Note that:

(1) $\operatorname{Faf}(G ; x, y)=0$, if $G$ has no perfect matchings.

(2) $\operatorname{Faf}(G ; x, y)=1$, if $G$ has a unique perfect matching or $G$ is an empty graph (with no vertices).

Consequently, we have the following conclusions. 
Corollary $2.3 \quad$ (1) $F(G, x)=\operatorname{Faf}(G ; x, 1)$.

(2) $\operatorname{Af}(G, x)=\operatorname{Faf}(G ; 1, x)$.

(3) The number of perfect matchings of $G$ is $\operatorname{Faf}(G ; 1,1)$.

Under the action of the automorphism group of $G$, all perfect matchings are divided into several equivalent classes [23]. By selecting a perfect matching from each equivalent class, we obtain the system of distinct representatives. Since each perfect matching in an equivalent class has the same forcing number and anti-forcing number, we have another formula to compute the di-forcing polynomial.

Lemma 2.4 Let $G$ be a graph with a perfect matching $M$. Let $\mathcal{R}(G)$ and $s(M)$ denote the system of distinct representatives of the perfect matching equivalent classes and the cardinality of the equivalent class containing $M$, respectively. Then

$$
F a f(G ; x, y)=\sum_{M \in \mathcal{R}(G)} s(M) x^{f(G, M)} y^{a f(G, M)} .
$$

\section{Computing the di-forcing polynomial}

Now, there are two ways to compute the di-forcing polynomial of a graph: One is the summation over all perfect matchings of a general graph $G$ basing on Definition 2.3; The other is the summation over all perfect matchings of the system of distinct representatives of the perfect matching equivalent classes for a high symmestry of graph $G$ basing on Lemma 2.4. Thus, we provide two methods to compute the forcing number or anti-forcing number of a given perfect matching $M$ : Exhaustive enumeration is relatively simple but time-consuming, while integer linear programming is effective.

Let $G$ be a graph with a perfect matching $M, n$ vertices, $m$ edges and $E(G)=$ $\left\{e_{1}, e_{2}, \ldots, e_{m}\right\}$. The symmetric difference of two finite sets $A$ and $B$ is $A \oplus B=(A \cup$ $B) \backslash(A \cap B)$.

\subsection{Exhaustive enumeration}

The basic idea of exhaustive enumeration is as follows: We generate all possible edge subsets from small to large one by one, until finding the first subset $S$ which is a forcing or anti-forcing set of $M$. Hence $S$ is a minimum forcing or anti-forcing set, and $|S|$ is the forcing or anti-forcing number of $M$. 
Although the exhaustive enumeration is successfully used to compute the forcing polynomials of $C_{60}, C_{70}, C_{72}[20,22,23,27]$, it is too time-consuming to compute anti-forcing polynomials of these graphs. When computing forcing number and anti-forcing number using this method, the total number of subsets to be verified are $\sum_{r=0}^{F(G)}\left(\begin{array}{c}\frac{n}{2} \\ r\end{array}\right)$ and $\sum_{r=0}^{A f(G)}\left(\begin{array}{c}m-\frac{n}{2} \\ r\end{array}\right)$, respectively. If $n$ and $m$ are large, then $\sum_{r=0}^{A f(G)}\left(\begin{array}{c}m-\frac{n}{2} \\ r\end{array}\right)$ is much larger than $\sum_{r=0}^{F(G)}\left(\begin{array}{l}\frac{n}{2} \\ r\end{array}\right)$, since the first one not only has large combinatorial numbers but also has more terms $(F(G) \leqslant A f(G) \leqslant(\Delta-1) F(G)[16$, Theorem 2.2]). For example, we use this method to compute the anti-forcing numbers of perfect matchings of $C_{60}$, where $n=60, m=90$ and $\sum_{r=0}^{20}\left(\begin{array}{c}60 \\ r\end{array}\right) \gg \sum_{r=0}^{10}\left(\begin{array}{c}30 \\ r\end{array}\right)$. After computing for consecutive six months on a personal computer, we only find the minimum anti-forcing sets of 75 representives with anti-forcing numbers 8,9 and 10 . However, to finish the work, we have to verify all subsets with size from 11 to 20 of 60 edges to find the first anti-forcing set of the remaining 83 representives, since $A f\left(C_{60}\right)=20=(3-1) F\left(C_{60}\right)$.

\subsection{Integer linear programming}

By Lemma 2.1, we can see that finding the minimum forcing set $S \subseteq M$ is an optimization problem: $S$ is a smallest edge subset containing at least one edge of each $M$-alternating cycle. Therefore, the forcing number of a perfect matching $M$ of graph $G$ can be computed as follows.

We generate all $M$-alternating cycles of $G$, such as collecting all connected components (each must be a $M$-alternating cycle) among all symmetric differences between $M$ and each of the other perfect matchings. Let $\mathbf{M}=\left(m_{i j}\right)$ be an $l \times m$ matrix, where $l$ is the number of $M$-alternating cycles, and each row vector $\left(m_{i 1}, m_{i 2}, \ldots, m_{i m}\right)$ of $\mathbf{M}$ represents the $0-1$ vector correesponding to an $M$-alternating cycle $c_{i}$, with 1 on the positions of the edges both in $M$ and $c_{i}$, and 0 otherwise, i.e. $m_{i j}=1$ if and only if $e_{j} \in c_{i} \cap M, i=1,2, \ldots, l, j=$ $1,2, \ldots, m$. Let $\mathbf{b}=(1,1, \ldots, 1)^{T}$ and $\mathbf{c}=(1,1, \ldots, 1)^{T}$ be two vectors with dimensions $l$ and $m$, respectively. Thus, we have the following integer linear programming (ILP):

$$
\begin{aligned}
& \text { (ILP): } \min \mathbf{c}^{T} \mathbf{x} ; \\
& \text { s.t. } \\
& \left\{\begin{array}{l}
\mathbf{M} \mathbf{x} \geqslant \mathbf{b}, \\
\mathbf{x} \in\{0,1\}^{m} .
\end{array}\right.
\end{aligned}
$$

Now, the optimal value of (ILP) is just the forcing number of $M$ and the optimal 
solution $\mathbf{x}$ is the incidence vector of a minimum forcing set $S$, i.e. $e_{i} \in S$ if and only if $x_{i}=1$.

\section{Remark 1}

(1) If we put 1 at the positions of the edges which are in $c_{i}$ but not in $M$, and 0 otherwise, i.e. $m_{i j}=1$ if and only if $e_{j} \in c_{i} \backslash M, i=1,2, \ldots, l, j=1,2, \ldots, m$ in M, then the above (ILP) gives the anti-forcing number of the perfect matching $M$. Thus we can compute the di-forcing polynomial of $G$ by Definition 2.3 or Lemma 2.4 .

(2) F. Zhang, G. Lin, M. Zheng [28] proposed a direct exhaustive searching method to obtain all $M$-alternating cycles of a graph.

(3) If we relax the condition $\mathbf{x} \in\{0,1\}^{m}$ to $\mathbf{x} \geq 0$ or $0 \leq \mathbf{x} \leq 1$, i.e. $0 \leq x_{j} \leq 1, j=$ $1,2, \ldots, m$, then we may obtain a fractional solution. For example, for the No. 187 perfect matching $M$ of $C_{60}$, we get a fractional optimal solution of anti-forcing number $\frac{27}{2} \leq a f\left(C_{60}, M\right)=14$ (see Figure 1 ).

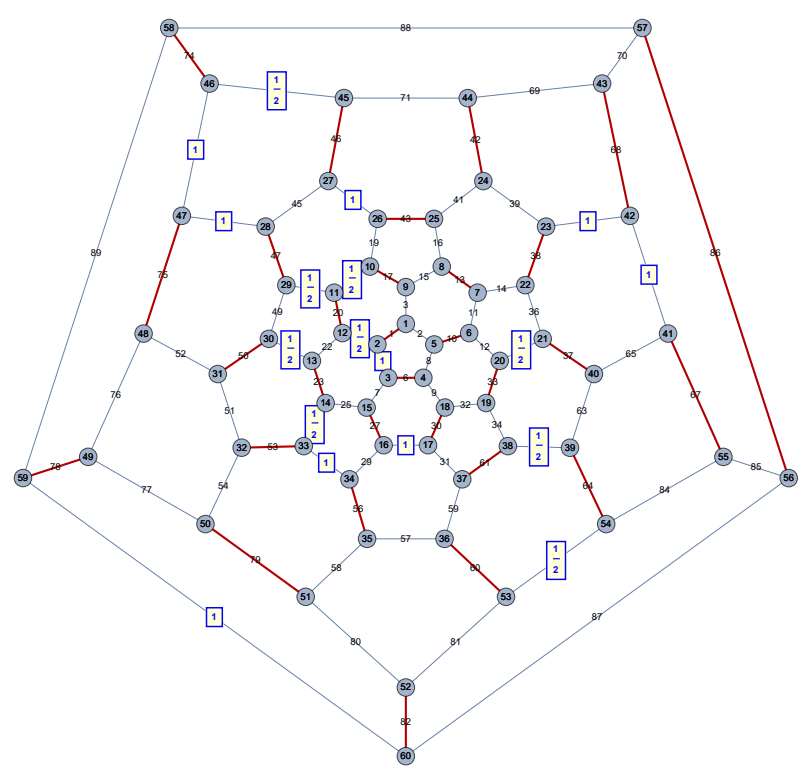

Figure 1. A fractional optimal solution 


\subsection{An example}

The cube graph $Q_{3}$ (see Figure 2) has 9 perfect matchings (see Figure 3, matching edges are thick). According to the symmetry of $Q_{3}$, they can be divided into two equivalent classes: $\left\{M_{1}, M_{2}, M_{3}\right\}$ and $\left\{M_{4}, M_{5}, M_{6}, M_{7}, M_{8}, M_{9}\right\}$. Let $M_{1}$ and $M_{4}$ be the representatives of these two equivalent classes, respectively.

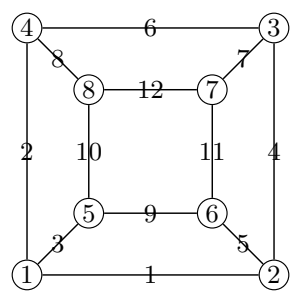

Figure 2. The cube graph $Q_{3}$

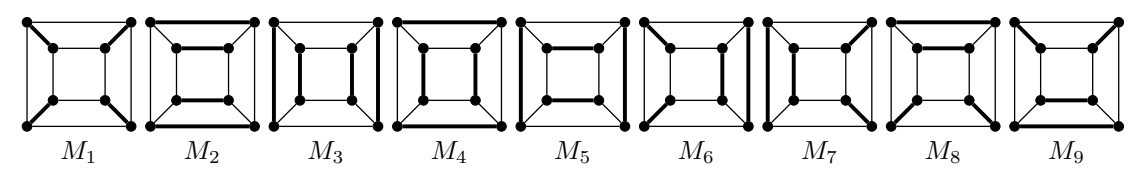

Figure 3. 9 perfect matchings of $Q_{3}$

Firstly, let's calculate the forcing numbers of $M_{1}$ and $M_{4}$.

By making symmetric differences of $M_{1}$ with each of the other perfect matchings, we obtain $6 M_{1}$-alternating cycles (see Figure 4).
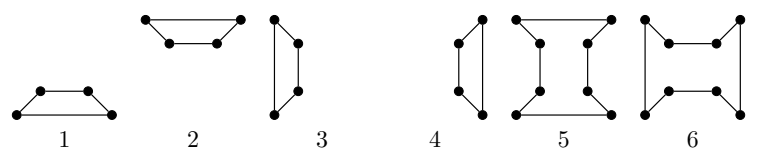

Figure 4. $M_{1}$-alternating cycles

Then the coefficient matrix $\mathbf{M}_{\mathbf{1}}$ w.r.t. $M_{1}, \mathbf{b}, \mathbf{c}$ and $\mathbf{x}$ of (ILP) are as follows:

$$
\begin{aligned}
\mathbf{M}_{\mathbf{1}}= & \left(\begin{array}{cccccccccccc}
0 & 0 & 1 & 0 & 1 & 0 & 0 & 0 & 0 & 0 & 0 & 0 \\
0 & 0 & 0 & 0 & 0 & 0 & 1 & 1 & 0 & 0 & 0 & 0 \\
0 & 0 & 1 & 0 & 0 & 0 & 0 & 1 & 0 & 0 & 0 & 0 \\
0 & 0 & 0 & 0 & 1 & 0 & 1 & 0 & 0 & 0 & 0 & 0 \\
0 & 0 & 1 & 0 & 1 & 0 & 1 & 1 & 0 & 0 & 0 & 0 \\
0 & 0 & 1 & 0 & 1 & 0 & 1 & 1 & 0 & 0 & 0 & 0
\end{array}\right) \\
\mathbf{b} & =(1,1,1,1,1,1)^{T},
\end{aligned}
$$




$$
\begin{gathered}
\mathbf{c}=(1,1,1,1,1,1,1,1,1,1,1,1)^{T} \\
\mathbf{x}=\left(x_{1}, x_{2}, x_{3}, x_{4}, x_{5}, x_{6}, x_{7}, x_{8}, x_{9}, x_{10}, x_{11}, x_{12}\right)^{T} .
\end{gathered}
$$

By solving the (ILP), we obtain $\mathbf{x}=(0,0,0,0,1,0,0,1,0,0,0,0)^{T}$. That is to say that $S=\left\{e_{5}, e_{8}\right\}$ is a minimum forcing set and the forcing number of $M_{1}$ is $\mathbf{c}^{T} \mathbf{x}=2$, the number of 1's in $\mathbf{x}$.

Similarly, the coefficient matrix $\mathbf{M}_{4}$ w.r.t. $M_{4}$ is

$$
\mathbf{M}_{4}=\left(\begin{array}{llllllllllll}
1 & 0 & 0 & 0 & 0 & 1 & 0 & 0 & 0 & 1 & 1 & 0 \\
0 & 0 & 0 & 0 & 0 & 0 & 0 & 0 & 0 & 1 & 1 & 0 \\
1 & 0 & 0 & 0 & 0 & 1 & 0 & 0 & 0 & 0 & 0 & 0 \\
1 & 0 & 0 & 0 & 0 & 1 & 0 & 0 & 0 & 1 & 1 & 0 \\
1 & 0 & 0 & 0 & 0 & 1 & 0 & 0 & 0 & 1 & 0 & 0 \\
1 & 0 & 0 & 0 & 0 & 1 & 0 & 0 & 0 & 0 & 1 & 0 \\
1 & 0 & 0 & 0 & 0 & 0 & 0 & 0 & 0 & 1 & 1 & 0 \\
0 & 0 & 0 & 0 & 0 & 1 & 0 & 0 & 0 & 1 & 1 & 0
\end{array}\right)
$$

By solving the (ILP), we obtain the forcing number of $M_{4}$ is $\mathbf{c}^{T} \mathbf{x}=2$.

Secondly, let's calculate the anti-forcing numbers of $M_{1}$ and $M_{4}$.

The new coefficient matrix $\mathbf{M}_{1}^{\prime}$ w.r.t. $M_{1}$ is

$$
\mathbf{M}_{1}^{\prime}=\left(\begin{array}{llllllllllll}
1 & 0 & 0 & 0 & 0 & 0 & 0 & 0 & 1 & 0 & 0 & 0 \\
0 & 0 & 0 & 0 & 0 & 1 & 0 & 0 & 0 & 0 & 0 & 1 \\
0 & 1 & 0 & 0 & 0 & 0 & 0 & 0 & 0 & 1 & 0 & 0 \\
0 & 0 & 0 & 1 & 0 & 0 & 0 & 0 & 0 & 0 & 1 & 0 \\
1 & 0 & 0 & 0 & 0 & 1 & 0 & 0 & 0 & 1 & 1 & 0 \\
0 & 1 & 0 & 1 & 0 & 0 & 0 & 0 & 1 & 0 & 0 & 1
\end{array}\right)
$$

By solving the (ILP), we obtain the anti-forcing number of $M_{1}$ is $\mathbf{c}^{T} \mathbf{x}=4$.

The new coefficient matrix $\mathbf{M}_{\mathbf{4}}^{\prime}$ w.r.t. $M_{4}$ is

$$
\mathbf{M}_{4}^{\prime}=\left(\begin{array}{llllllllllll}
0 & 0 & 1 & 0 & 1 & 0 & 1 & 1 & 0 & 0 & 0 & 0 \\
0 & 0 & 0 & 0 & 0 & 0 & 0 & 0 & 1 & 0 & 0 & 1 \\
0 & 1 & 0 & 1 & 0 & 0 & 0 & 0 & 0 & 0 & 0 & 0 \\
0 & 1 & 0 & 1 & 0 & 0 & 0 & 0 & 1 & 0 & 0 & 1 \\
0 & 0 & 1 & 1 & 0 & 0 & 0 & 1 & 0 & 0 & 0 & 0 \\
0 & 1 & 0 & 0 & 1 & 0 & 1 & 0 & 0 & 0 & 0 & 0 \\
0 & 0 & 1 & 0 & 1 & 0 & 0 & 0 & 0 & 0 & 0 & 1 \\
0 & 0 & 0 & 0 & 0 & 0 & 1 & 1 & 1 & 0 & 0 & 0
\end{array}\right)
$$

By solving the (ILP), we obtain the anti-forcing number of $M_{4}$ is $\mathbf{c}^{T} \mathbf{x}=3$.

Finally, by the above arguments and Lemma 2.4, the di-forcing polynomial of cube graph $Q_{3}$ is

$$
\operatorname{Faf}\left(Q_{3} ; x, y\right)=3 x^{2} y^{4}+6 x^{2} y^{3}
$$




\section{Applications}

As applications of (ILP), we obtain di-forcing polynomials, and particularly anti-forcing polynomials, of $C_{60}, C_{70}$ and $C_{72}$ (See Figure $5[12,20,22,29]$ ).
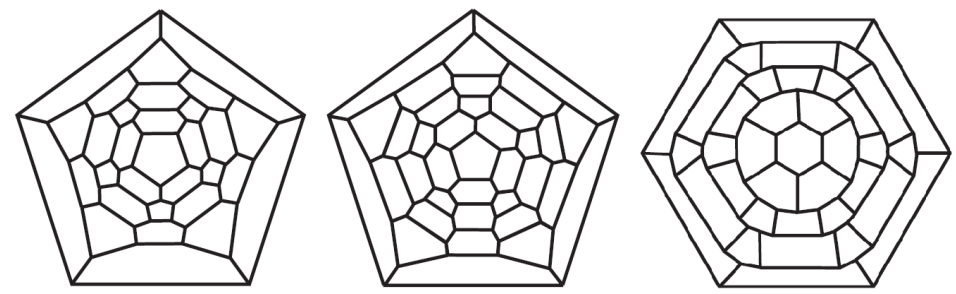

Figure 5. $C_{60}, C_{70}$ and $C_{72}$

\subsection{The di-forcing polynomial of $C_{60}$}

In 1985, H. Kroto, J. Heath, S. O'Brien, R. Curl, R. Smalley found $C_{60}$ [12], an important member of Fullerenes. Vukičević, Harry W. Kroto, and Milan Randić [23-25] solved the forcing number problem of $C_{60}$. Using the automorphism group of $C_{60}$, they divided 12500 perfect matchings into 158 equivalent classes, and gave the forcing number of each representative. By Lemma 2.4, their result is equivalant to the forcing polynomial of $C_{60}$.

By Lemma 2.4 and using the function "LinearProgramming" with option "Integers" to solve the (ILP) in software Mathematica, we compute the di-forcing polynomial of $C_{60}$.

Theorem 4.1 The di-forcing polynomial of $C_{60}$ is

$$
\begin{aligned}
\text { Faf }\left(C_{60} ; x, y\right)= & x^{10} y^{20}+20 x^{9} y^{18}+60 x^{9} y^{16}+100 x^{8} y^{16}+612 x^{8} y^{14} \\
& +60 x^{7} y^{14}+376 x^{8} y^{13}+30 x^{7} y^{13}+640 x^{8} y^{12}+1080 x^{7} y^{12} \\
& +60 x^{6} y^{12}+325 x^{8} y^{11}+2290 x^{7} y^{11}+420 x^{6} y^{11}+20 x^{8} y^{10} \\
& +600 x^{7} y^{10}+1720 x^{6} y^{10}+660 x^{5} y^{10}+886 x^{6} y^{9}+1280 x^{5} y^{9} \\
& +30 x^{6} y^{8}+1230 x^{5} y^{8} .
\end{aligned}
$$

Particularly, we have a new result as follows:

Corollary 4.2 The anti-forcing polynomial of $C_{60}$ is

$$
\begin{aligned}
A f\left(C_{60}, x\right)= & F a f\left(C_{60} ; 1, x\right)=x^{20}+20 x^{18}+160 x^{16}+672 x^{14}+406 x^{13} \\
& +1780 x^{12}+3035 x^{11}+3000 x^{10}+2166 x^{9}+1260 x^{8} .
\end{aligned}
$$


Similarly, we obtain the following known results.

Corollary 4.3 (1) [24,27] The forcing polynomial of $C_{60}$ is

$$
\begin{aligned}
F\left(C_{60}, x\right)= & F a f\left(C_{60} ; x, 1\right)=x^{10}+80 x^{9}+2073 x^{8}+4060 x^{7}+3116 x^{6} \\
& +3170 x^{5} .
\end{aligned}
$$

(2) [15] The number of perfect matchings of $C_{60}$ is

$$
\operatorname{Faf}\left(C_{60} ; 1,1\right)=12500 .
$$

\subsection{The di-forcing polynomial of $C_{70}$}

$C_{70}$ has 52168 perfect mathings that are divided into 2780 equivalent classes [20]. Similar to the above calculation procedure of $C_{60}$, we obtain the di-forcing polynomial of $C_{70}$.

Theorem 4.4 The di-forcing polynomial of the $C_{70}$ is

$$
\begin{aligned}
\text { Faf }\left(C_{70} ; x, y\right)= & 2 x^{11} y^{21}+20 x^{11} y^{19}+40 x^{10} y^{19}+10 x^{9} y^{18}+10 x^{11} y^{17} \\
& +300 x^{10} y^{17}+290 x^{9} y^{17}+90 x^{10} y^{16}+30 x^{9} y^{16}+171 x^{8} y^{16} \\
& +230 x^{10} y^{15}+1614 x^{9} y^{15}+1000 x^{8} y^{15}+20 x^{10} y^{14} \\
& +1100 x^{9} y^{14}+1731 x^{8} y^{14}+290 x^{7} y^{14}+1490 x^{9} y^{13} \\
& +4130 x^{8} y^{13}+2480 x^{7} y^{13}+320 x^{9} y^{12}+4220 x^{8} y^{12} \\
& +7230 x^{7} y^{12}+360 x^{6} y^{12}+90 x^{9} y^{11}+1120 x^{8} y^{11} \\
& +7040 x^{7} y^{11}+4950 x^{6} y^{11}+40 x^{8} y^{10}+1320 x^{7} y^{10} \\
& +6360 x^{6} y^{10}+660 x^{5} y^{10}+50 x^{7} y^{9}+1880 x^{6} y^{9} \\
& +1300 x^{5} y^{9}+40 x^{6} y^{8}+140 x^{5} y^{8} .
\end{aligned}
$$

Consequently, we have a new result as follows:

Corollary 4.5 The anti-forcing polynomial of $C_{70}$ is

$$
\begin{aligned}
A f\left(C_{70}, x\right)= & F a f\left(C_{70} ; 1, x\right)=2 x^{21}+60 x^{19}+10 x^{18}+600 x^{17} \\
& +291 x^{16}+2844 x^{15}+3141 x^{14}+8100 x^{13}+12130 x^{12} \\
& +13200 x^{11}+8380 x^{10}+3230 x^{9}+180 x^{8} .
\end{aligned}
$$

Also, we obtain the following known results. 
Corollary 4.6 (1) [20,27] The forcing polynomial of $C_{70}$ is

$$
\begin{aligned}
F\left(C_{70}, x\right)= & F a f\left(C_{70} ; x, 1\right)=32 x^{11}+680 x^{10}+4944 x^{9}+12412 x^{8} \\
& +18410 x^{7}+13590 x^{6}+2100 x^{5} .
\end{aligned}
$$

(2) [20] The number of perfect matchings of $C_{70}$ is

$$
\operatorname{Faf}\left(C_{70} ; 1,1\right)=52168
$$

Remark 2 Zeng [27] corrects the corresponding computation result in [20], see [29].

\subsection{The di-forcing polynomial of $C_{72}$}

$C_{72}$ has 77400 perfect mathings that are divided into 3470 equivalent classes [22]. Similar to the above calculation procedure of $C_{60}$, we obtain the di-forcing polynomial of $C_{72}$.

Theorem 4.7 The di-forcing polynomial of the $C_{72}$ is

$$
\begin{aligned}
\operatorname{Faf}\left(C_{72} ; x, y\right)= & x^{12} y^{24}+24 x^{11} y^{22}+76 x^{11} y^{20}+168 x^{10} y^{20} \\
& +1120 x^{10} y^{18}+244 x^{9} y^{18}+276 x^{10} y^{17}+24 x^{9} y^{17} \\
& +1464 x^{10} y^{16}+2935 x^{9} y^{16}+55 x^{8} y^{16}+696 x^{10} y^{15} \\
& +2504 x^{9} y^{15}+537 x^{8} y^{15}+372 x^{10} y^{14}+7622 x^{9} y^{14} \\
& +1684 x^{8} y^{14}+456 x^{7} y^{14}+7836 x^{9} y^{13}+3000 x^{8} y^{13} \\
& +3274 x^{7} y^{13}+2764 x^{9} y^{12}+3338 x^{8} y^{12}+8538 x^{7} y^{12} \\
& +764 x^{6} y^{12}+108 x^{9} y^{11}+570 x^{8} y^{11}+781 x^{7} y^{11} \\
& +6822 x^{6} y^{11}+12 x^{8} y^{10}+1470 x^{7} y^{10}+7225 x^{6} y^{10} \\
& +432 x^{5} y^{10}+48 x^{7} y^{9}+1983 x^{6} y^{9}+948 x^{5} y^{9} \\
& +15 x^{6} y^{8}+177 x^{5} y^{8} .
\end{aligned}
$$

Especially, we have a new result as follows:

Corollary 4.8 The anti-forcing polynomial of $C_{72}$ is

$$
\begin{aligned}
\text { Af }\left(C_{72}, x\right)= & \text { Faf }\left(C_{72} ; 1, x\right)=x^{24}+24 x^{22}+244 x^{20}+1364 x^{18}+300 x^{17} \\
& +4454 x^{16}+3737 x^{15}+10134 x^{14}+14110 x^{13}+15404 x^{12} \\
& +15318 x^{11}+9139 x^{10}+2979 x^{9}+192 x^{8}
\end{aligned}
$$

Also, we obtain the following known results. 
Corollary 4.9 (1) [22] The forcing polynomial of $C_{72}$ is

$$
\begin{aligned}
F\left(C_{72}, x\right)= & F a f\left(C_{72} ; x, 1\right)=x^{12}+100 x^{11}+4096 x^{10}+24037 x^{9} \\
& +9196 x^{8}+21604 x^{7}+16809 x^{6}+1557 x^{5}
\end{aligned}
$$

(2) [22] The number of perfect matchings of $C_{72}$ is

$$
\operatorname{Faf}\left(C_{72} ; 1,1\right)=77400
$$

\subsection{The di-forcing polynomials of other fullerenes}

We have obtained all di-forcing polynomials of other fullerenes with carbon atoms at most 60 (including all 1812 isomers of $C_{60}$ ). A detailed discussion of these results will be presented in other articles.

\section{References}

[1] H. Abeledo, G. W. Atkinson, Unimodularity of the Clar number problem, Lin. Algebra Appl. 420 (2007) 441-448.

[2] H. Abeledo, G. W. Atkinson, The Clar and Fries problems for benzenoid hydrocarbons are linear programs, in: P. Hansen, P. Fowler, M. Zheng (Eds.), Proceedings of the DIMACS Workshop on Discrete Mathematical Chemistry, Am. Math. Soc., Providence, 2000, pp. 1-8.

[3] P. Adams, M. Mahdian, E. S. Mahmoodian, On the forced matching numbers of bipartite graphs, Discr. Math. 281 (2004) 1-12.

[4] P. Afshani, H. Hatami, E. S. Mahmoodian, On the spectrum of the forced matching number of graphs, Australas. J. Comb. 30 (2004) 147-160.

[5] Z. Che, Z. Chen, Forcing on perfect matchings - a survey, MATCH Commun. Math. Comput. Chem. 66 (2011) 93-136.

[6] E. Clar, The Aromatic Sextet, Wiley, London, 1972.

[7] K. Deng, S. Liu, X. Zhou, Forcing and anti-forcing polynomials of perfect matching of a pyrene system, MATCH Commun. Math. Comput. Chem. 85 (2021) 27-46.

[8] K. Deng, H. Lü, T. Wu, Forcing and anti-forcing polynomials of a polyomino graph, arXiv: 2012.12898 (2020).

[9] K. Fries, Uber Byclische Verbindungen und ihren Vergleich mit dem Naphtalin, Ann. Chem. 454 (1927) 121-324. 
[10] F. Harary, D. J. Klein, T. P. Živković, Graphical properties of polyhexes: Perfect matching vector and forcing, J. Math. Chem. 6 (1991) 295-306.

[11] H. Hwang, H. Lei, Y. Yeh, H. Zhang, Distribution of forcing and anti-forcing numbers of random perfect matchings on hexagonal chains and crowns, 2015. http://140. 109.74.92/hk/?p=873.

[12] H. W. Kroto, J. R. Heath, S. C. O'Brien, R. F. Curl, R. Smalley, $C_{60}$ : Buckminsterfullerene, Nature 318 (1985) 162-163.

[13] D. J. Klein, M. Randić, Innate degree of freedom of a graph, J. Comput. Chem. 8 (1987) 516-521.

[14] D. J. Klein, V. Rosenfeld, Forcing, freedom, and uniqueness in graph theory and chemistry, Croat. Chem. Acta. 87 (2014) 49-59.

[15] D. J. Klein, T. G. Schmalz, G. E. Hite, W. A. Seitz, Resonance in $C_{60}$ buckminsterfullerene, J. Am. Chem. Soc. 108 (1986) 1301-1302.

[16] H. Lei, Y. Yeh, H. Zhang, Anti-forcing numbers of perfect matchings of graphs, Discr. Appl. Math. 202 (2016) 95-105.

[17] P. Hansen, M. Zheng, The Clar number of a benzenoid hydrocarbon and linear programming, J. Math. Chem. 15 (1994) 93-107.

[18] X. Li, Hexagonal systems with forcing single edges, Discr. Appl. Math. 72 (1997) 295-301.

[19] M. Randić, D. J. Klein, Kekulé valence structures revisited, Innate degrees of freedom of $\pi$-electron couplings, in: N. Trinajstić (Eds.), Mathematics and Computational Concepts in Chemistry, Ellis Horwood, Chichester, 1986, pp. 274-282.

[20] M. Randić, D. Vukičević, Kekulé structures of fullerene $C_{70}$, Croat. Chem. Acta. 79 (2006) 471-481.

[21] M. E. Riddle, The minimum forcing number for the torus and hypercube, Discr. Math. 245 (2002) 283-292.

[22] D. Vukičević, I. Gutman, M. Randić, On instability of fullerene $C_{72}$, Croat. Chem. Acta. 79 (2006) 429-436.

[23] D. Vukičević, H. W. Kroto, M. Randić, Atlas of Kekulé valence structures of buckminsterfullerene, Croat. Chem. Acta. 78 (2005) 223-234.

[24] D. Vukičević, M. Randić, On Kekulé structures of buckminsterfullerene, Chem. Phys. Lett. 401 (2005) 446-450. 
[25] D. Vukičević, M. Randić, Detailed atlas of Kekulé structures of the buckminsterfullerene, in: F. Cataldo, A. Graovac, O. Ori (Eds.), The Mathematics and Topology of Fullerenes, Springer, Dordrecht, 2011, pp. 153-169.

[26] D. Vukičević, N. Trinajstić, On the anti-forcing number of benzenoids, J. Math. Chem. 42 (2007) 575-583.

[27] L. Zeng, The computation of perfect matchings and forcing numbers of fullerenes (Chinese), Bachelor Thesis, Lanzhou Univ., Lanzhou, 2009.

[28] F. Zhang, G. Lin, M. Zheng, A method for the all perfect matchings (Chinese), J. Xinjiang Univ. 4 (1987) 1-8.

[29] H. Zhang, S. Zhao, R. Lin, The forcing polynomial of catacondensed hexagonal systems, MATCH Commun. Math. Comput. Chem. 73 (2015) 473-490.

[30] S. Zhao, H. Zhang, Anti-forcing polynomials for benzenoids systems with forcing edges, Discr. Appl. Math. 250 (2018) 342-356.

[31] S. Zhao, H. Zhang, Forcing and anti-forcing polynomials of perfect matchings for some rectangle grids, J. Math. Chem. 57 (2019) 202-225. 